\title{
Redo hepatic artery reconstruction for thrombosis can save grafts and patients without retransplantation: lessons learned from 1,355 adult living donor liver transplantations
}

\author{
Su Young Hong ${ }^{1}$, Nam-Joon Yi ${ }^{1}$, Jeong-Moo Lee ${ }^{1}$, Suk Kyun Hong ${ }^{1}$, YoungRok Choi ${ }^{1}$, Ung Sik Jin ${ }^{2}$, Hak Chang ${ }^{2}$, Kwang- \\ Woong Lee ${ }^{1}$, Kyung-Suk Suh${ }^{1}$, Kyung Won Minn²
}

\footnotetext{
${ }^{1}$ Division of Hepatobiliary and Pancreatic Surgery, Department of Surgery, Seoul National University Hospital, Seoul, Korea

${ }^{2}$ Department of Plastic and Reconstructive Surgery, Seoul National University Hospital, Seoul, Korea
}

Background: Hepatic artery thrombosis (HAT) after liver transplantation is associated with a marked increase in morbidity, being the main cause of graft loss and bile duct complication leading patients' deaths. Retransplantation is often unavailable in most Asian countries due to donor organ shortage. Herein, we evaluated the outcome of patients with HAT after adult living donor liver transplantation (ALDLT) under aggressive surgical correction strategy.

Methods: From January 2000 to June 2019, 1,355 recipients underwent ALDLT in Seoul National University Hospital. Surgical redo reconstruction for HAT was applied in every case except the evidence of graft necrosis or late detection (since postoperative day 60 ) of HAT. Median follow-up period was 89 months. Survival outcomes and the rates of biliary complication of patients with HAT were compared with others without HAT.

Results: Postoperative HAT was developed in 33 cases (2.4\%) at a median time of 3.5 days (range, 1-82 days). Overall graft survival rates were lower in patients with HAT $(84.8 \%)$ than others without HAT $(98.0 \%)(\mathrm{P}<0.001)$. However, patient survival rates were similar between two groups $(72.7 \%$ vs. $83.8 \%, \mathrm{P}=0.115)$. Biliary complication rates were higher in patients with HAT $(54.5 \%)$ than others without HAT (32.0\%) ( $P=0.008)$. Among 33 patients with HAT, 30 patients $(90.9 \%)$ underwent redo arterial reconstruction. The technical success rate of redo reconstruction was $83.3 \%(n=25)$. After redo-reconstruction, three patients $(10.0 \%)$ underwent retransplantation and $76.6 \%$ of patients $(n=23)$ were finally survived. Another three patients with HAT underwent conservative management $(n=2)$ and retransplantation directly $(n=1)$.

Conclusions: HAT after ALDLT was associated with increased rates of biliary complication and significantly attenuates graft survival outcome. However, aggressive surgical treatment can save the graft in $90 \%$ without retransplantation and patient survival was not affected.

Corresponding author: Nam-Joon Yi

E-mail: gsleenj@hanmail.net

(C) The Korean Society for Transplantation

This is an Open Access article distributed under the terms of the Creative Commons Attribution Non-Commercial License (http://creativecommons.org/licenses/by-nc/4.0/) which permits unrestricted non-commercial use, distribution, and reproduction in any medium, provided the original work is properly cited. 